\title{
"Transversalidad" de la transversalidad. Análisis de una estrategia didáctica aplicada a la educación para la sostenibilidad
}

\author{
Pedro Álvarez Suárez \\ Universidade de Granada, Espanha \\ Pedro Vega Marcote \\ Universidade de A Coruña, Espanha
}

\begin{abstract}
Resumo
Al comprar, tanto sencillos productos de primera necesidad como artículos de lujo, estamos, en la mayoría de los casos, tomando decisiones que afectan a la calidad ambiental del planeta. Pero son pocas las personas que conocen la relación entre nuestros hábitos cotidianos y la actual crisis ambiental global. Así, aunque muchas personas están concienciadas para actuar sosteniblemente, no saben cómo hacerlo la mayoría de las veces. Por ello, es imprescindible que se les de a los ciudadanos - consumidores - una información adecuada y se les capacite para actuar sosteniblemente y, en ello, dos áreas transversales, la Educación Ambiental y la Educación para el Consumo juegan un papel fundamental. De acuerdo con ello, se presenta una estrategia didáctica que, desde la "transversalidad" de la transversalidad, pretende concienciar y capacitar hacia un consumo responsable y sostenible, de la cual se muestran, además, los resultados obtenidos tras su aplicación en alumnos de educación secundaria (14-16 años).
\end{abstract}

Palabras clave

Transversalidad; Estrategia didáctica; Sostenibilidad

\section{Transversalizar las transversales}

Al igual que en otros países europeos, la introducción de las áreas transversales (áreas transdisciplinares, en otros países) en el diseño curricular español (Ley Orgánica General del Sistema Educativo, 1990), 
pretendía que el alumnado completaran su educación en las materias tradicionales con nuevos conocimientos - conceptuales, procedimentales y actitudinales - que les prepararan para su integración social como ciudadanos responsables, solidarios y críticos, capaces, además, de actuar individual y colectivamente en la protección del medio ambiente, evitar la discriminación sexual y xenofóbica, promocionar hábitos de salud y consumo sostenible, etc.

Pero, el desarrollo curricular de estas nuevas áreas no sólo implica la incorporación de sus objetivos al Proyecto de Centro y, consecuentemente el compromiso de todos los componentes del mismo, sino, además, la flexibilización de espacios y horarios y la reestructuración de los contenidos de las restantes áreas "tradicionales" para dar cabida a estos nuevos contenidos. Todo ello dificulta su implantación, aduciéndose falta de tiempo o espacio en los programas educativos o desconocimiento de las materias por parte del profesorado (Yus, 1996) e, incluso, la ausencia de marcos teóricos que orienten su desarrollo (Trímboli, 2004).

Una de las características de los temas transversales es su elevado contenido actitudinal, lo que conlleva que algunas de estas áreas guarden estrechas relaciones entre sí. Así, por ejemplo, los contenidos relativos a la alimentación pueden relacionarse con la Educación para el consumo (qué comprar, dónde comprar, información del etiquetado, publicidad...), con la Educación para la Salud (repercusiones sobre la misma de diversos hábitos alimenticios....), con la Educación Ambiental (residuos producidos por los envases, impactos de la producción, como contaminación agraria difusa, escasez de recursos hídricos....), y con la Educación para la Paz (multinacionales, mercado internacional....); por su parte, el Worldwatch Institute, en su publicación sobre El Estado del Mundo, asocia la Educación del consumidor (la sociedad de consumo) con la Educación Ambiental (aumento de residuos y de la huella ecológica) con la Educación para la Salud (aumento de enfermedades) y con la Educación para la Ciudadanía (disminución del compromiso cívico) (Gadner, Assadourian y Sarin, 2004); y también la UNESCO, en el Plan de Aplicación de la Década de las Naciones Unidas para el Desarrollo Sostenible, señala que la educación para la sostenibilidad gira en torno de la sociedad, la economía y el medio ambiente (UNESCO, 2005), ejes que están íntimamente relacionados con el consumo

Partiendo de este hecho se propone una estrategia de enseñanzaaprendizaje que trabaje las áreas transversales con una perspectiva más 
amplia, que implique referencias claras y continuas hacia su integración en el sentido de transversalizar las transversales. Es decir, se propone una estrategia didáctica para trabajar con estas áreas que proporcione no sólo los contenidos conceptuales propios de ellas, sino también sensibilidad y sentido crítico, junto con las habilidades que capaciten al alumnado para la acción. Se trata, en definitiva, de que el alumnado, ciudadanos del futuro, desarrollen la estrategia ecológica de la acción (Morín, 1995) como modelo de intervención en el medio, estableciendo una estrecha relación entre pensamiento y acción.

En el caso concreto del ejemplo incluido para ilustrar su desarrollo (Contaminación mental), el alumnado, previo conocimiento del tema y sensibilización acerca de sus repercusiones, deben capacitarse para actuar, individual y colectivamente, frente a la presión social (publicidad, modas...) que empuja al consumismo, causa, a su vez, de importantes deterioros ambientales y, en ocasiones de nuestra propia salud (caso de la publicidad de tabaco, alcohol, de multinacionales de comidas rápidas...) y de la discriminación económica de determinados sectores o grupos tanto en nuestro entorno como en numerosos lugares del planeta.

\section{Ejemplificación de la estrategia didáctica: contaminación mental}

\section{Fundamentación teórica}

Consumo y medio ambiente. Una amistad peligrosa

El consumo se ha adueñado de todo y el consumismo como pulsión parece ser el motor de la sociedad. La sociedad de consumo, llamada por algunos sociedad del bienestar y de la riqueza, induce a la adquisición y acumulación de cosas.

Para mostrar como funciona la sociedad de consumo en la que estamos inmersos, Galbraith (2002) acuñó la expresión "la noria de la producción", comparándola a una especie de "jaula gigante para hamsters", en la que todos estamos dentro sin poder o querer salir de ella, y donde corremos cada vez más deprisa para seguir en el mismo sitio. Esta noria, cuyos múltiples giros por minuto lo hacen en torno al eje del consumo, ha creado una lamentable situación en lo que respecta a la sostenibilidad del planeta, pues depende esencialmente de una tecnología intensiva en energía 
y capital, conduciendo, por tanto, a un agotamiento más rápido de los recursos naturales - energéticos y no energéticos -, y a la producción de cantidades crecientes de residuos que son arrojados al entorno, causando el espectacular aumento del grado de toxicidad ambiental detectado en los últimos cincuenta años. Tendencia que no parece que vaya a invertirse, sino que, por el contrario aumenta progresivamente el gasto de materias primas y energía para responder a las crecientes demandas del sector consumo, originando lo que Broswimmer (2005) denomina ecocidio. Pues ello causa múltiples problemas de carácter ambiental, social, político y cultural que ponen en riesgo la propia supervivencia de la Tierra.

Sin embargo, quizás sea la ecocrisis en que nos hayamos inmersos la que mejor ha contribuido a esclarecer la irracionalidad de los modelos vigentes de producción-consumo y los estilos de desarrollo insostenible que caracterizan las sociedades de los países desarrollados, pues, como indicaba Commoner (1979), "la degradación del medio ambiente es una consecuencia directa del modo de producción y de la forma de producir" y, consecuentemente, del modo de consumir.

Pero este consumismo sin sentido promueve un modelo insostenible ambiental y socialmente. Ambientalmente porque si el modelo de consumo de los países del norte se extendiera por todo el mundo, serían necesarios 3 planetas (con sus materias primas, fuentes energéticas...) para atender la demanda. $\mathrm{Y}$ socialmente porque sólo un $12 \%$ de la gente que vive en Norteamérica y Europa occidental es responsable del 60\% de ese consumo, mientras que los que viven en el sudeste asiático o en el África subsahariana representan sólo un 3,2\%, por ello es necesario un compromiso internacional pues existe una responsabilidad compartida pero diferenciada (GEO-4, UNEP, 2007).

Aunque, el consumo en sí no es un valor negativo. Todos los seres humanos requerimos de un nivel de consumo específico para suplir nuestras necesidades. El consumo es una cualidad intrínseca al ser humano; pero la pasión por el consumo - el llamado consumismo y, también, "consumerismo" -, por el contrario, es una manifestación patológica de la sociedad contemporánea. El consumismo ha creado una sociedad en la cual sin duda la gente disfruta de muchas más comodidades que sus ancestros. Pero en el debe se incluyen los costes sociales y ambientales, pues al comprar tanto 
sencillos productos de primera necesidad como artículos de lujo, estamos, en la mayoría de los casos, tomando decisiones que afectan a la calidad ambiental de nuestro planeta. Sin embargo, son pocas las personas que conocen la relación entre nuestros hábitos cotidianos y la crisis ambiental global en que estamos inmersos. Por ejemplo, pocos relacionan el tomar una hamburguesa con la destrucción de los bosques tropicales (la instalación de grandes granjas para el ganado, cuya producción cárnica se destina en gran parte a productos de comida rápida, ha provocado enormes deforestaciones en países como Brasil y Costa Rica).

Podemos afirmar, sin temor a equivocarnos, que la mayoría de los problemas de degradación ambiental se originan en fenómenos socioeconómicos determinados por estilos de vida y modos de comportamiento (producción-consumo). El movimiento Lifestyle está absolutamente en lo cierto cuando pide a la gente "vivir sencillamente para que sencillamente otros puedan vivir". La cuestión está clara: o se subordina el consumo a la satisfacción de las demandas humanas o, por el contrario, se cede a las tendencias consumistas y se subordina a ello el sistema cultural.

Cada acto de consumo contribuye a transformar el entorno, por tanto, los consumidores son un factor clave para, cambiando su forma de actuación, redefinir un modelo de sociedad que contribuya a la sostenibilidad.

\section{"Contaminación mental"}

El sistema económico imperante en la sociedad actual se basa en un aumento constante del consumo (obviando, por supuesto, sus repercusiones ambientales), lo que requiere cauces específicos de persuasión de los potenciales consumidores. El trabajo, el ocio y la vida privada están manipulados y orientados hacia un consumo programado, gratificante y controlado, que es constantemente alimentado por los medios de comunicación. El papel fundamental de los medios de comunicación es el desarrollar la sociedad de consumo. Debido a su capacidad de difusión y a su enorme influencia se han convertido en un instrumento privilegiado para dirigir y controlar las demandas de la población. Sin publicidad disminuiría el consumo y esto afectaría negativamente a las economías. Ésta es la razón por la cual los mensajes publicitarios nos invaden, nos asfixian.... y nos 
manipulan. A través de publicidad los fabricantes intentan informar sobre sus productos, crear necesidades a los individuos, imponer modas, introducir nuevos productos $y$, en definitiva, orientar a los distintos grupos hacia el consumo continuo, no sólo manteniendo el consumo necesario sino aumentando intensamente el consumo superfluo.

En la sociedad actual la publicidad constituye un instrumento privilegiado para adaptar la demanda de bienes de consumo a las condiciones y exigencias del sistema productivo. Nada resulta más elocuente que el desenfrenado aumento de las inversiones en publicidad, sobre todo en los países desarrollados, en los que un ciudadano medio recibe más de una incitación o mensaje consumista por minuto. $Y$ todos ellos resultan de alguna manera condicionantes de la conducta.

Según Guerin, el aire que respiramos es un compuesto de oxígeno, nitrógeno y publicidad. La publicidad lo invade todo y nos incita machaconamente a adquirir productos, por lo que enlazando con lo expresado en el apartado anterior respecto a los efectos negativos del consumo sobre el medio ambiente, estamos totalmente de acuerdo con Araujo (1993) cuando afirma que "el acto más peligroso para la naturaleza es conectar el televisor". Pues, mientras que, en España, se calcula que el precio de venta de un diario sólo cubre el $30 \%$, aproximadamente, de su coste real, financiándose el $70 \%$ restante con la publicidad. La financiación publicitaria es total, en cambio, en el caso de las emisoras de radio comerciales y televisiones privadas.

Por otra parte, la publicidad crea profundas contradicciones por sus diversos efectos sobre la sociedad; contradicciones que también afectan al Estado en cuanto que éste recibe importantes beneficios económicos de ciertas pautas de consumo cuya publicidad es muy criticada por generar graves problemas de salud (tabaco y alcohol). Un ejemplo paradigmático de esta contradicción es uno de los tótems de la sociedad de consumo: el automóvil. Todos giramos en torno a este elemento consustancial a la sociedad de consumo. Elemento que, es bien conocido, provoca miles de muertes y degrada de forma muy importante el medio (contaminación urbana, efectos sobre el cambio climático, agotamiento de recursos, lluvias ácidas...), pero que también recauda directa e indirectamente miles de millones para el estado a través de los impuestos, tanto directos (de matriculación, circulación, 
sanciones...), como indirectos (sobre el carburante, IVA de reparaciones, seguros...). Antítesis que pone de manifiesto la profunda imbricación y consolidación del sistema; es decir, la casi imposibilidad de modificarlo profundamente sin alterar sus elementos económicos.

\section{El papel de la educación}

Por ello, el Estado apuesta por modificar otros elementos de la sociedad, como, por ejemplo, la educación. Pero, reformar la educación no implica reformar la sociedad, es un simple maquillaje de una parte de esa sociedad para que otros elementos no cambien. Las reformas educativas no son sino nuevos intentos de legitimar la ideología dominante. Su eficacia educativa es, por tanto, muy relativa, pero al menos debe servir para poner de manifiesto estas contradicciones y concienciar a los jóvenes acerca de ellas.

Sin embargo, mucha gente de los países industrializados - los mayores consumidores - está concienciada para actuar sosteniblemente, el problema es que este interés discurre en paralelo con un sentimiento de impotencia y una incapacidad percibida para concretarlo en comportamientos concretos (Jensen, 1993; Uzzel, Rutland y Whistance, 1995).

Debemos, por tanto, potenciar actitudes vigilantes ante la publicidad y los discursos persuasivos sobre consumo. La educación debe evitar que la publicidad "disminuya las defensas" ante el consumo superfluo, lo que puede lograr dotando al consumidor, a los millones de consumidores, de una conciencia crítica ante el consumo, dándoles una información adecuada y capacitándolos para tomar decisiones al respecto que, a su vez, se traduzcan en acciones acordes con un estilo de vida más sostenible, promoviendo un consumo sostenible (racional y ecoselectivo).

Es en este contexto donde cobra todo su sentido la que denominamos Educación para la Sosteniblilidad, que debe involucrar íntegramente a las personas en el tratamiento de problemas reales y concretos, sin conformarse con la mera discusión de posibles soluciones (Vega, Álvarez, Freitas y Fleurí, 2007). Los individuos no sólo deben saber valorar sino que deben estar capacitados para actuar a favor de la sostenibilidad, frente a la insuficiencia de desarrollar solamente hábitos de buen ciudadano, más próximos en muchas ocasiones a la urbanidad (no dejar basuras en el entorno natural...) que a una auténtica preocupación y a unas actuaciones sostenibles. 
Desde esta perspectiva hemos desarrollado un modelo didáctico "experimental" de carácter investigativo y base constructivista con el que pretendemos que estudiantes de educación secundaria (14-16 años) adquieran los conceptos, actitudes y conductas necesarios para propiciar unas actuaciones más cercanas a lo que se ha dado en llamar desarrollo sostenible, capacitándolos para participar, individual y colectivamente, en la solución de problemáticas socioambientales de su entorno próximo, sin perder la perspectiva de lo global, y que no son sino la consecuencia de nuestra forma de vida (Vega y Álvarez, 2006).

\section{Estrategia para trabajar la transversalidad de la transversalidad}

La estrategia didáctica propuesta tiene como eje fundamental el planteamiento y trabajo con problemáticas socioambientales del entorno próximo del alumnado. Con su desarrollo esperábamos conseguir que los alumnos que la sigan logren aprendizajes conceptuales significativos acerca del tópico tratado, desarrollen actitudes y habilidades ("saber hacer") para actuar a favor de la sostenibilidad, mejorando su autoestima al respecto. Factores que, junto con la norma subjetiva determinan la intención de actuar, precedente inmediato de una conducta sostenible (Eagly y Chayken, 1992; Leclerq, 1992).

Las diferentes fases del modelo, que pueden verse en la figura siguiente (Figura 1), requieren para su desarrollo una serie de etapas que deben estar integradas en la secuencia actividades de aprendizaje (Álvarez y Vega, 2007). 


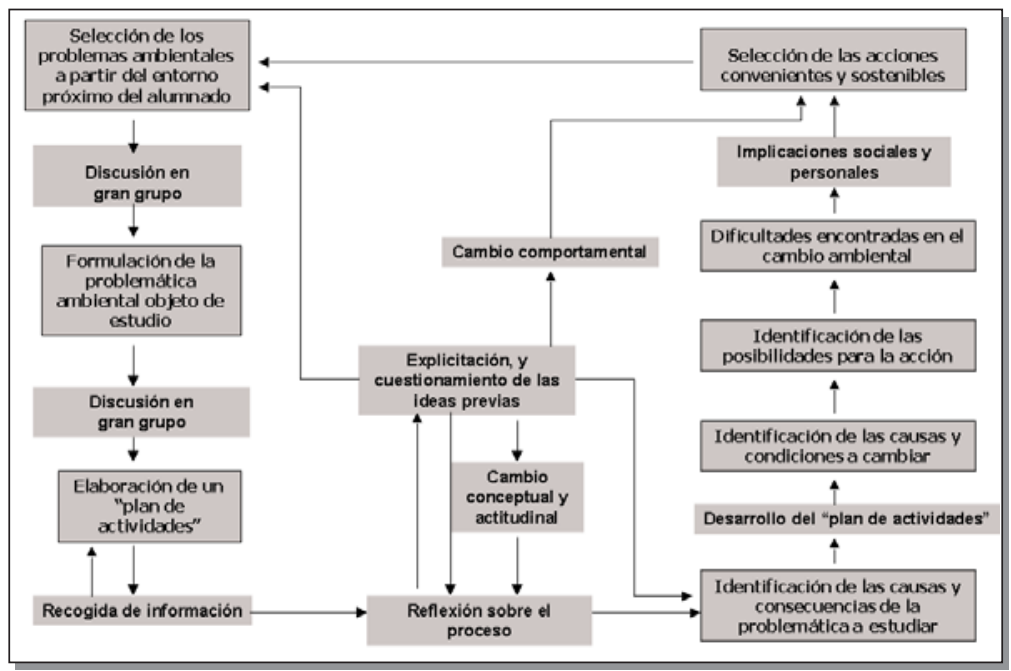

Figura 1 - Modelo didáctico para el desarrollo de competencias en la acción

El trabajo se realizaba en grupos que periódicamente eran coordinados en reuniones de gran grupo (ver cuadro 1). La función del profesorado ha sido la de director-coordinador del proceso de aprendizaje de los alumnos, orientando el trabajo de cada uno de los grupos, coordinando las puestas en común y proporcionando información cuando ha sido necesario.

\section{Cuadro 1 - Organización del trabajo de los alumnos}

\begin{tabular}{|c|c|c|c|}
\hline \multicolumn{4}{|c|}{ Organización del trabajo de los alumnos } \\
\hline Formas de agrupamiento & Tamaño del grupo & & Criterios de agrupamiento \\
\hline Grupo fijo & 73 alumnos & - & Grupos de aula $(29+23+21)$ \\
\hline Grupo flexible & $4 / 5$ alumnos & & $\begin{array}{l}\text { Naturaleza de la tarea } \\
\text { Nivel de conocimientos } \\
\text { Objetivos que se persiguen } \\
\text { Posibilidades organizativas } \\
\text { Capacidad de expresión } \\
\text { Uniformidad en el aprendizaje } \\
\text { Relaciones entre el alumnado } \\
\text { Relaciones con el profesorado }\end{array}$ \\
\hline
\end{tabular}




\section{Análisis y evaluación de la estrategia didáctica}

\section{Método}

De acuerdo con la perspectiva constructivista asumida para la estrategia didáctica que queremos evaluar, debemos conocer la situación inicial del alumnado, en cuanto a conocimientos conceptuales, actitudes ambientales e intención de realizar conductas sostenibles, no sólo como punto de partida de sus aprendizajes, sino también como referencia para evaluar el cambio conceptual, actitudinal y comportamental (intención de conducta) que esta estrategia produce en ellos.

\section{Participantes}

Los participantes en la investigación tenían la siguiente procedencia (Cuadro 2):

Cuadro 2 - Procedencia de los participantes

\begin{tabular}{|c|c|c|c|}
\hline CENTRO & GRUPO & $\begin{array}{c}\text { OP. BIOLOGíA Y } \\
\text { GEOLOGÍA } \\
\text { (no de alumnos) }\end{array}$ & $\begin{array}{c}\text { OP. HUMANIDADES } \\
\text { (no de alumnos) }\end{array}$ \\
\hline $\begin{array}{c}\text { IES "ÁNGEL GANIVET" } \\
\text { (Granada, España) }\end{array}$ & A & 29 & - \\
\hline & B & 10 & 13 \\
\hline & C & - & 21 \\
\hline
\end{tabular}

Las características personales (género y edad) de los participantes se reflejan en el siguiente cuadro (Cuadro 3).

\section{Cuadro 3 - Características personales de los participantes (edad y} género)

\begin{tabular}{|c|c|c|c|c|}
\cline { 2 - 4 } \multicolumn{1}{c|}{} & Mujeres & Varones & Edad Media & Desviación típica \\
\hline Recuento (N) & 41 & 32 & & \\
\hline$\%$ & $56,16 \%$ & $43,84 \%$ & 14,923 & 0,422 \\
\hline
\end{tabular}




\section{Material y procedimiento}

La comprobación del funcionamiento del modelo de intervención ha constado de las siguientes fases:

1. Pretest: Al comienzo del curso académico se administró a la totalidad de los participantes un dossier que constaba de:

a) una escala de actitudes ambientales, previamente construida y validada (Álvarez, De la Fuente, García y Fernández, 2002);

b) un cuestionario para conocer su formación previa sobre el medio ambiente y problemas socioambientales, fuentes de información y educación "formal" recibida al respecto. Con ambos cuestionarios se pretendía conocer los "errores conceptuales" que, previamente a la instrucción, pueden tener los alumnos en cuanto a conceptos relativos al medio ambiente, la problemática socioambiental y el desarrollo sostenible, con el doble objetivo de programar las actividades de aprendizaje en función de ellos, para reconducir las ideas erróneas, y establecer un nivel de referencia para comprobar la eficacia de la estrategia en lo referente al campo conceptual deseado;

c) dos cuestionarios sobre conceptos relacionados con el medio ambiente:

c.1. uno con diez preguntas abiertas, para así, poder conocer los conocimientos de partida, previamente validado (Álvarez, De la Fuente, Perales y García, 2002), que se había construido a partir del elaborado por la British Ecological Society en 1987 (Cherret, 1989), añadiéndole varios ítems relacionados con la acción antrópica sobre el medio en función de su "popularidad", es decir, teniendo en cuenta la frecuencia con que aparecen en los medios de comunicación;

c.2. el otro incluía diversos problemas socioambientales que los alumnos debían ordenar en función la importancia (gravedad) atribuida a cada uno de ellos. Ambos se incluyen en el anexo 1 como Conocimientos-1 y Conocimientos-2, respectivamente;

d) una escala de intención de realizar conductas sostenibles, elaborada ex profeso para esta investigación (anexo 2); 
e) la escala de deseabilidad social de Marlone y Crowe (1960), traducida y adaptada al castellano por Ávila y Tomé (1989), con el fin de controlar los posibles sesgos que este factor pudiera introducir en las propuestas de los alumnos.

2. Tratamiento experimental: los contenidos se impartieron siguiendo la estrategia didáctica experimental (antes descrita), con la que se pretendía lograr su capacitación para la acción a favor de la sostenibilidad.

3. Postest: al finalizar el curso, se administró de nuevo el dossier a los participantes a fin de comprobar si se habían producido diferencias en las actitudes hacia la sostenibilidad, conocimientos conceptuales e intención de conducta y, en su caso, si estas eran significativas a nivel estadístico.

El tiempo empleado en la administración de las encuestas ha sido de una hora, tanto en el pretest como en el postest. Las instrucciones proporcionadas a los alumnos en cada pasación fueron idénticas en todos los grupos.

\section{Análisis estadísticos y resultados}

Los datos recogidos fueron analizados con el paquete estadístico SPSS (Statistical Package for the Social Sciences), versión 12.0 y Excel 2000.

En primer lugar, analizamos si existían diferencias estadísticamente significativas en cuanto a las variables personales y variables dependientes entre los tres grupos ( $\mathrm{A}, \mathrm{B}$ y $\mathrm{C}$ ) en el pretest, para determinar si pueden ser considerados como un solo grupo, obteniéndose los siguientes resultados (Tabla 1).

Tabla 1 - Homogeneidad de los grupos al inicio de la experiencia

\begin{tabular}{|l|c|c|c|c|}
\hline \multicolumn{1}{|c|}{ Variable } & Prueba realizada & "t" & Chi-cuadrado & Sig. \\
\hline Edad & "t" de Student & $-0,087$ & & 0,931 \\
Género & Prueba de contingencia & & 0,201 & 0,654 \\
Formación previa & "t" de Student & $-0,127$ & & 0,899 \\
Conocimientos conceptuales & "t" de Student & 1,009 & & 0,315 \\
Actitudes ambientales & "t" de Student & $-1,617$ & & 0,109 \\
Intención de conducta & "t" de Student & $-0,558$ & & 0,512 \\
\hline
\end{tabular}


El análisis descriptivo de los datos obtenidos en el pretest tiene un doble interés, pues, por una parte nos permitirá conocer el nivel de conocimientos conceptuales de los alumnos al comienzo del curso y, por otra parte, nos permitirá la contrastación del cambio conceptual, actitudinal y comportamental producido, en su caso, al finalizar el curso.

Los estadísticos descriptivos acerca de los conocimientos conceptuales sobre el medio ambiente, problemáticas ambientales y sostenibilidad (Cuestionario 1), muestran que, en su mayor parte, los sujetos desconocían los conceptos y estructuras conceptuales relacionadas con el medio ambiente, a pesar de la incidencia que muchos de ellos han tenido y tienen en los medios de comunicación, siendo por tanto bastante bajas las puntuaciones obtenidas, aunque ligeramente superiores para los del grupo A (véase la tabla 2), debido, posiblemente, a estar constituido exclusivamente por alumnado de la opción Ciencias.

Tabla 2 - Conocimientos conceptuales sobre medio ambiente y problemáticas ambientales (Conocimientos 1), en el pretest. Estadísticos descriptivos

\begin{tabular}{|c|c|c|c|c|}
\hline Grupos & Participantes (N) & Puntuación media & $\begin{array}{c}\text { Desviación } \\
\text { típica }\end{array}$ & Error típico de la media \\
\hline A & 29 & 3,833 & 1,02992 & 0,13296 \\
\hline B y C & 44 & 3,541 & 1,78425 & 0,28571 \\
\hline
\end{tabular}

Para contrastar las posibles diferencias entre los conocimientos conceptuales sobre medio ambiente entre los grupos B y C antes del tratamiento experimental (pretest), se realizó la prueba de Mann-Whitney" sobre los porcentajes de respuestas acertadas por ambos grupos, comprobándose que no existían diferencias estadísticamente significativas entre ellos (valor $=47,00 ; p<0.821$ ); lo que se reafirmó mediante un test sobre la igualdad de medias en muestras independientes (la " $t$ " de Student, obteniéndose como resultados: $t=0,183 ; \mathrm{p}=0,000$ ).

En cuanto a la importancia (gravedad) atribuida a diversos problemas ambientales (cuestionario "Conocimientos 2"), las valoraciones son muy similares en todos los grupos, destacando la tendencia a dar mayor 
importancia (considerarlas más graves) las problemáticas del entorno próximo de los sujetos (atención "a lo local") frente a otras de carácter planetario (atención "a lo global").

Respecto a las actitudes hacia el medio ambiente los datos obtenidos en el pretest, también reflejan resultados similares para todos los grupos: 43,6001 con una desviación típica $=10,45604$ para el grupo $\mathrm{A}$ y 42,931, con una desviación típica $=9,82849$, para el conjunto de los grupos B y C; además, al realizar el contraste entre los grupos mediante un análisis de varianza (ANOVA), éste mostró que no existían diferencias significativas entre las medias de los grupos $(F=8,419 ; p=0,000)$. Por otra parte, cabe destacar que los valores obtenidos son bastante altos, lo que podría ser debido al alto nivel de sensibilización que los españoles muestran hacia el medio ambiente, aunque no podemos descartar que dado el tipo de instrumento utilizado, una escala tipo Likert, los sujetos hayan sobrevalorado esta variable por sentirse partícipes de un proyecto singular ("efecto Hawthorne").

En cuanto a la intención de realizar conductas sostenibles la puntuación media obtenida por los grupos es muy similar: 35,3500 sobre 100 (desviación típica $=3,93517$ ) para el grupo A y 35,8462 (desviación típica $=3,97054$ ) para el conjunto de los los grupos B y C. Pudimos pues concluir que los grupos considerados en la investigación no presentan en el pretest diferencias estadísticamente significativas en ninguna de las variables personales y variables dependientes consideradas.

Una vez terminada la experiencia (al finalizar el curso académico), con los datos recogidos en el postest se realizaron análisis estadísticos para comprobar si los participantes mejoraron significativamente, en términos estadísticos, sus actitudes ambientales, sus conocimientos conceptuales acerca del medio ambiente y su intención de realizar acciones sostenibles.

Así, en cuanto a conocimientos conceptuales sobre el medio (Cuestionario 1), todos los sujetos obtuvieron puntuaciones notablemente superiores a las conseguidas en el pretest; si bien, como puede verse en la siguiente tabla, fueron más altas para el grupo $\mathrm{A}$. 
Tabla 3 - Conocimientos conceptuales sobre medio ambiente

(Conocimientos 1) en el postest. Estadísticos descriptivos

\begin{tabular}{|c|c|c|c|c|}
\hline Grupo & Participantes (N) & Puntuación media & Desviación típica & Error típico de la media \\
\hline A & 29 & 8,0833 & 1,04435 & 0,17029 \\
\hline B y C & 44 & 6,9744 & 1,06344 & 0,16392 \\
\hline
\end{tabular}

Por su parte, el contraste de medias para cada uno de los grupos entre el pretest y el postest, muestra diferencias estadísticamente significativas ( $t=$ $10,874 ; p=0,000)$.

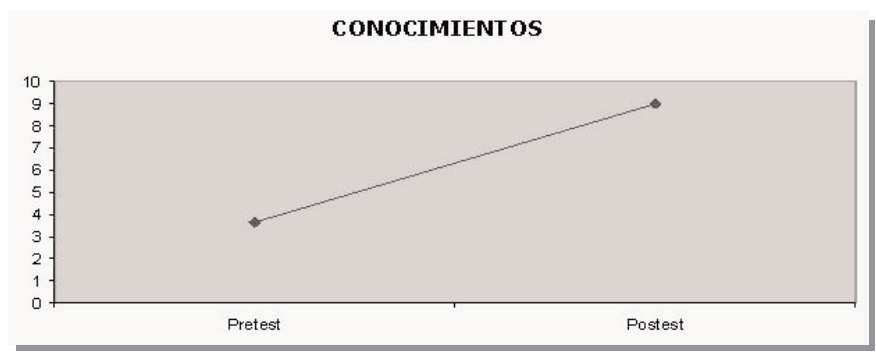

Figura 2 - Evolución de los conocimientos conceptuales sobre medio ambiente y problemáticas ambientales

Sin embargo, las pruebas "a posteriori" (DHS de Tukey y prueba de Scheffe) realizadas sobre los grupos B y C, manifiestan diferencias entre ambos, en el sentido de que mientras los alumnos de la opción Ciencias (del grupo B) aumentan notablemente sus conocimientos (porcentaje de respuestas acertadas $=65,53 \%$ ), los alumnos de la opción Humanidades (grupo C y parte del B) obtienen resultados poco mejores que los obtenidos en el pretest (porcentaje de respuestas acertadas $=49,67 \%$ ).

Respecto a la valoración de la importancia (gravedad) de las problemáticas socioambientales recogidas en el cuestionario "Conocimientos 2", debemos resaltar, por una parte, la tendencia de los componentes del grupo A a considerar más importantes los problemas de carácter "global", mientras que los sujetos del grupo $\mathrm{C}$ apenas han variado el sentido de su valoración respecto al pretest; por otra parte, la práctica totalidad de los participantes $(62,9 \%)$ consideren que los incendios forestales son uno de los problemas ambientales más importantes. 
En lo referente a las actitudes hacia el medio ambiente, el contraste entre el grupo A y el conjunto de los grupos B y C a partir de los datos obtenidos en el postest, indica que las diferencias entre ambos grupos no son estadísticamente significativas (ver tabla 4).

Tabla 4 - Prueba de muestras independientes pretest-postest de los grupos (Actitudes)

\begin{tabular}{|c|c|c|c|c|c|c|c|c|c|}
\hline \multirow[t]{3}{*}{ Grupos } & \multicolumn{2}{|c|}{$\begin{array}{l}\text { Prueba de } \\
\text { Levene para la } \\
\text { igualdad de } \\
\text { varianzas }\end{array}$} & \multicolumn{7}{|c|}{ Prueba $T$ para la igualdad de medias } \\
\hline & \multirow[t]{2}{*}{$\mathrm{F}$} & \multirow[t]{2}{*}{ Sig. } & \multirow[t]{2}{*}{$t$} & \multirow[t]{2}{*}{$\mathrm{Gl}$} & \multirow[t]{2}{*}{$\begin{array}{c}\text { Sig. } \\
\text { (bilateral) }\end{array}$} & \multirow[t]{2}{*}{$\begin{array}{l}\text { Diferencia } \\
\text { de medias }\end{array}$} & \multirow[t]{2}{*}{$\begin{array}{l}\text { Error tip. de } \\
\text { la diferencia }\end{array}$} & \multicolumn{2}{|c|}{$\begin{array}{c}95 \% \text { intervalo de confianza } \\
\text { para la diferencia }\end{array}$} \\
\hline & & & & & & & & inferior & superior \\
\hline ByC & 26,315 & 0,000 & $-23,670$ & 49,284 & 0,000 & $-35,93333$ & 1,63134 & $-43,07548$ & $-31,89118$ \\
\hline A & 27,267 & 0,000 & $-22,316$ & 46,583 & 0,000 & $-37,07692$ & 1,66142 & $-40,92005$ & $-33,73380$ \\
\hline
\end{tabular}

Pero, los datos también reflejan que, a pesar del alto nivel constatado en el pretest, se ha producido un aumento en el nivel actitudinal de los sujetos, siendo las diferencias entre ambos momentos estadísticamente significativas (véase la figura 3). Para esta variable, las pruebas "a posteriori" realizadas indicaron que no existen diferencias significativas entre los grupos B y C; cuyas respuestas, por otra parte, son similares a las del conjunto de la población andaluza (Junta de Andalucía, 2004).

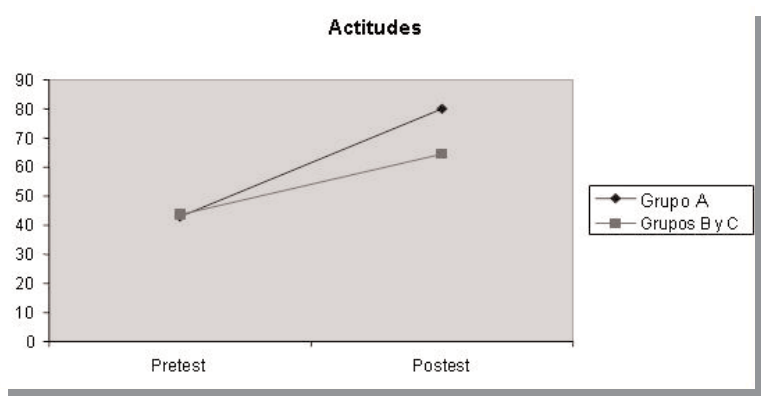

Figura 3 - Evolución de las actitudes hacia el medio ambiente (pretestPostest) 
Con los datos recogidos sobre la intención de realizar conductas sostenibles en el pretest y en el pretest, hemos realizado los estadísticos descriptivos (Tabla 5) y el test "t" de Student (Tabla 6).

Tablas 5 - Estadísticos pretest-postest grupos experimental/control (intención de conducta)

\begin{tabular}{|c|c|c|c|c|c|}
\hline Grupos & \multicolumn{2}{|c|}{ N } & $\begin{array}{c}\text { Media } \\
\text { general }\end{array}$ & Desviación típica & Error típico de la media \\
\hline \multirow{2}{*}{ B y C } & Pretest & 44 & 35,3500 & 3,93517 & 0,50803 \\
\cline { 2 - 6 } & Postest & 44 & 43,9500 & 3,54218 & 0,58639 \\
\hline \multirow{2}{*}{ A } & Pretest & 29 & 35,8462 & 3,97054 & 0,63580 \\
\cline { 2 - 6 } & Postest & 29 & 44,5385 & 2,80832 & 0,44969 \\
\hline
\end{tabular}

Tablas 6 - Prueba de muestras independientes pretest-postest grupo (intención de conducta)

\begin{tabular}{|c|c|c|c|c|c|c|c|c|c|}
\hline \multirow[t]{3}{*}{ Grupos } & \multicolumn{2}{|c|}{$\begin{array}{l}\text { Prueba de Levene } \\
\text { para la igualdad } \\
\text { de varianzas }\end{array}$} & \multicolumn{7}{|c|}{ Prueba $\mathrm{T}$ para la igualdad de medias } \\
\hline & \multirow[t]{2}{*}{$\mathrm{F}$} & \multirow[t]{2}{*}{ Sig. } & \multirow[t]{2}{*}{$\mathrm{t}$} & \multirow[t]{2}{*}{ GI } & \multirow[t]{2}{*}{$\begin{array}{c}\text { Sig. } \\
\text { (bilateral) }\end{array}$} & \multirow[t]{2}{*}{$\begin{array}{l}\text { Diferencia } \\
\text { de medias }\end{array}$} & \multirow[t]{2}{*}{$\begin{array}{l}\text { Error tip. de } \\
\text { la diferencia }\end{array}$} & \multicolumn{2}{|c|}{$\begin{array}{c}95 \% \text { intervalo de } \\
\text { confianza para la } \\
\text { diferencia }\end{array}$} \\
\hline & & & & & & & & inferior & superior \\
\hline By C & 2,630 & 0,096 & $-11,040$ & 88 & 0,000 & $-9,1000$ & 0,77585 & 12,43640 & $-6,63640$ \\
\hline A & 2,511 & 0,117 & $-11,162$ & 76 & 0,000 & $-8,69231$ & 0,77875 & $-10,24333$ & $-7,14129$ \\
\hline
\end{tabular}

Se puede observar entre el pretest y postest, la existencia de diferencias estadísticamente significativas, lo que determina que la intención de realizar conductas sostenibles ha aumentado (fig. 4). 


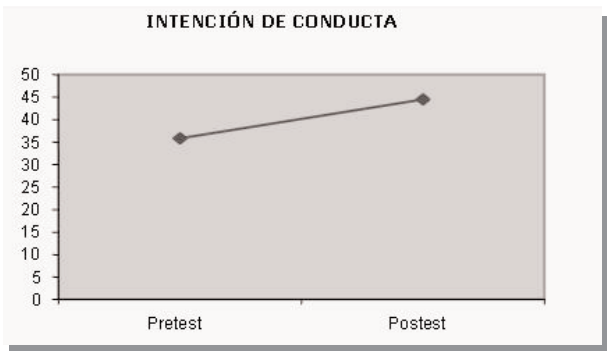

Figura 4 - Evolución de la intención de conducta hacia el medio (pretest-postest)

\section{Conclusiones}

La estrategia didáctica se revela como un recurso de gran eficacia para lo que denominamos Educación para la Sostenibilidad - al menos en los niveles correspondientes a la educación secundaria - , por cuanto que favorece el desarrollo de las actitudes proambientales de los alumnos y ello, a pesar de que el alto nivel actitudinal inicial que presentaban dejaba un reducido margen para el cambio.

Debemos también resaltar, por una parte, la tendencia a considerar más importantes los problemas de carácter "global", variando el sentido de su valoración respecto al pretest; por otra, el hecho de que una mayoría significativa de los participantes $(62,9 \%)$ consideren que los incendios forestales son uno de los problemas ambientales más importantes, coincidiendo con lo recogido por el Ecobarómetro de Andalucía 2004 (Junta de Andalucía, 2004).

En cuanto a "intención de conducta", las diferencias respecto al pretest se manifiestan, sobre todo, en comportamientos individuales, en el ahorro energético y de consumo responsable. Aspecto muy significativo, ya que la sostenibilidad desde el punto de vista práctico, implica que las personas desarrollen conductas proambientales sin que ello se considere estar haciendo algo excepcional.

Por otra parte, aunque no figuraba como objetivo de nuestro estudio y, por tanto, este hecho no ha sido cuantificado, hemos podido comprobar como durante el desarrollo de la experiencia los sujetos han superado la reticencia 
inicial hacia una forma de trabajo más activa, que les exigía un esfuerzo intelectual y procedimental mayor que la simple "toma de apuntes" o el uso del libro de texto a que estaban habituados.

Resulta pues muy recomendable que la transversalización de la transversalidad se haga a través de problemas socioambientales próximos a los alumnos y planteados por las actividades funcionales de éstos.

Destacamos, por último, que el adecuado desarrollo de la estrategia propuesta necesita, lógicamente, un desarrollo temporal más amplio que el preciso para la metodología expositiva; por ello, consideramos que se podrían incluir en los diseños de las actuaciones formativas fases no presenciales.

\section{Referências}

ALVAREZ, Pedro; DE LA FUENTE, Emilia I.; FERNÁNDEZ, María J. \& GARCíA, Juan (2002). Evaluación de actitudes ambientales en la ESO. Análisis de un instrumento. Alambique, no 22, pp. 77-86.

ÁlvareZ, Pedro; DE LA FUENTE, Emilia I.; PERALES, F. Javier \& GARCíA, Juan (2002). Analysis of a quasi-experimental design based on environmental problem-solving for the inicial training for future teachers of environmental education. The Journal of Environmental Education, vol. 33, no 2, pp. 19-21.

ÁLVAREZ, Pedro \& VEGA, Pedro (2008). Sustainable development and Environmental Education an inescapable relationship. Bulletin IAPS (International for PeopleEnvironment Studies), no 32, pp. 7-10.

ARAUJO, Joaquín (1993). Contaminación Mental. Málaga: Instituto de Investigaciones Ecológicas.

ÁVILA, Alejandro \& TOMÉ, M. Carmen (1989). Evaluación de la Deseabilidad Social y correlatos defensivos y emocionales. In A. Echevarría \& D. Páez (Eds.), Emociones: Perspectivas Psicosociales. Madrid: Fundamentos, pp. 505-514.

BROSWIMMER, Franz (2005). Ecocidio. Pamplona: Laetoli.

CAMPBELL, Donald \& STANLEY, Julian (1982). Diseños Experimentales y Cuasiexperimentales en la Investigación Social. Buenos Aires: Amorrourto.

CHERRETT, Joseph M. (1989): Key concepts: the results of a survey of our members opinions. In J. M. Cherrett (ed.), Ecological Concepts. Oxford: Blackwell, pp. 116.

COMMONER, Barry (1979). The Politics of Energy. New York : Knopf.

EAGLY, Alice H. \& CHAIKEN, Shelly (1993). The Psychology of Attitudes. Orlando: H.B.J. College Publishers.

GARDNER, Gary; ASSADOURIAN, Erik \& SARIN, R. (2004). L'estat del Consum Avui. En L'estat del Món. Barcelona: Worldwatch Institute. 
GALBRAITH, John K. (2002). How to get the poor of our consciencie. [En línea] [Acceso el 6 de febrero de 2009, disponible en http://www. sunset.backbone.olemiss.edu/ jmitchell/class/poor.htm].

GEO-4. (2007). Global Environnement Outlook. Valletta, Malta: United Nations Environment Programe (UNEP).

JENSEN, Bjarne Brunn (1993). The Concepts of Action and Action Competence. Comunicación presentada en el First International Workshop on "Children as Catalysts of Global Environmental Change. University of Surrey, 3-4 March, 1993.

JUNTA DE ANDALUCÍA (2004). Ecobarómetro de Andalucía 2004. Informe de síntesis de los resultados más relevantes. Sevilla: Consejería de Medio Ambiente de la Junta de Andalucía.

LECLERCQ, Denis (1992). Les facteurs de production de la conduite en matiére de santé. Colloque "Aprendre et vivre la santé à l'Ecole". Liége, 2-3, avril 1992.

MORIN, Edgard (1995). Una política de civilización. Complejidad, no 1.

TRÍMBOLI, Joan (2004). Educación del consumidor. Realidad y perspectivas. Consumer's International. [En línea] [Acceso el 20 de febrero de 2009, disponible en http://cinsumidoresint.org].

UNESCO (2005). Década de las Naciones Unidas para el Desarrollo Sostenible. Plan de Aplicación Internacional. París: UNESCO.

UZZELL, David L.; RUTLAND, Alisson \& WHISTANCE, Dan (1995). Questioning values in environmental education. In Y. Guerrier; N. Alexander; J. Chase \& M. O'Brien, Values and the Environment . Chichester: Wiley, pp. 172-182.

VEGA, Pedro \& ÁlVAREZ, Pedro (2006). Fondement et étude d'une stratégie méthodologique visant une éducation orientée vers la durabilité. Vertigo: la Revue Électronique en Sciences de L'environnement, vol. 7, no 3, pp. 1-17.

VEGA, Pedro; FREITAS, Mario; ÁLVAREZ, Pedro \& FLEURI, Reinaldo (2007). Marco teórico y metodológico de educación ambiental e intercultural para un desarrollo sostenible. Rev. Eureka. Enseñanza y Divugación Científica, vol. 4, № 3, pp. 539-554.

YUS, Rafael (1996). Hacia una Educación Global desde la Transversalidad. Madrid: Anaya. 
"TRANSVERSALIDADE" DA TRANSVERSALIDADE. ANÁLISE DE UMA ESTRATÉGIA DIDÁCTICA APLICADA À EDUCAÇÃO PARA A SUSTENTABILIDADE

\section{Resumo}

Ao comprar, tanto simples produtos de primeira necessidade como artigos de luxo, estamos, na maioria das vezes, a tomar decisões que afetam a qualidade ambiental do planeta. Entretanto, são poucas as pessoas que conhecem a relação entre os nossos hábitos cotidianos e a actual crise ambiental global. Assim, ainda que muitas pessoas estejam conscientes da necessidade de actuar sustentavelmente não sabem como fazê-lo na maioria das ocasiões. Por isso, é imprescindível que se dê aos cidadãos consumidores - uma informação adequada que os capacite para actuar sustentavelmente. Neste âmbito, duas áreas transversais, a Educação Ambiental e a Educação para o Consumo, exercem um papel fundamental. De acordo com isso, apresenta-se uma estratégia didáctica que, a partir da "transversalidade" da transversalidade, pretende consciencializar e capacitar para um consumo responsável e sustentável, do qual se mostram, além disso, os resultados obtidos após sua aplicação em alunos da educação secundária (14-16).

Palavras-chave

Transversalidade; Estratégia didática; Sustentabilidade 
"CROSS-CUTTING" OF CROSS-CUTTING. ANALYSIS OF AN APPLIED DIDACTIC STRATEGY IN EDUCATION FOR SUSTAINABILITY

\begin{abstract}
As consumers of both essential goods and luxury articles, we constantly make decisions which impact the environment. However, few people are aware of the relationship between their daily behaviour and the current global environmental crisis. Although many citizens are conscious of the need to behave appropriately to achieve sustainable development, they often do not know how to act in an environmentally friendly way. It is therefore essential that consumers be given appropriate information and be empowered to behave with environmental awareness. Environmental education for a sustainable future combined with consumer awareness as a vital role to play in this respect. To this end, a teaching strategy has been developed which bring together both perspectives. This strategy intends to raise environmental awareness and to empower people in relation to responsible consumption and sustainable development. It as also produced positive results among students of secondary education (14-16).
\end{abstract}

Key-words

Cross-cutting activity; Didactic strategy; Sustainability

Recebido em Março/2009

Aceite para publicação em Março/2010 


\section{ANEXO 1}

\section{Cuestionario de "Conocimientos 1"}

Responda brevemente a las siguientes cuestiones:

¿Qué entiendes por medio ambiente?

¿Qué es la ecología?

¿Qué entiendes por contaminación?

¿Qué es para tí el desarrollo sostenible?; ¿lo consideras posible?, ¿por qué ?

¿Qué es la "huella ecológica"

¿Qué es el agujero de ozono?

¿Qué entiendes por efecto invernadero y qué consecuencias puede tener?

¿Qué es la "regla de las tres R"

¿Qué es la biodiversidad?

¿Qué entiendes por educación ambiental?

\section{Cuestionario de "Conocimientos 2"}

Evaluar, de 0 a 10, cada una de las siguientes problemáticas ambientales, en función de su gravedad (incidencia negativa sobre el medio ambiente)

\begin{tabular}{|c|c|}
\hline Problemática & Evalua ción \\
\hline Agotamiento de los recursos naturales no renovables & \\
\hline Av ance progresivo de los desiertos & \\
\hline Contaminación acústica (ruido ambiental) & \\
\hline Contaminación de la hidrosfera por "mareas negras" (escapes de petroleo en el mar) & \\
\hline Contaminación en las ciudades (contaminación atmosférica, ruido...) & \\
\hline $\begin{array}{l}\text { Contaminación radioactiva (por accidentes en centrales nucleares, posibles conflictos bélicos, } \\
\text { pruebas nucleares, accidentes de submarinos y baroos nucleares....) }\end{array}$ & \\
\hline Defor estación de la selva amazónica (el "pulmón de la Tierra") & \\
\hline Deterioro de la oz ono sfera (agujero (s) de la capa de ozono) & \\
\hline El cambio climático global (consecuencia del "efecto invernadero") & \\
\hline Es casez de agua potable en numerosas zonas de la Tierra & \\
\hline Es casez de alimentos en muchas regiones del planeta & \\
\hline Explosión demográfica (rápido crecimiento de la población mundial) & \\
\hline Ex tinción de especies y pérdida de recursos energéticos & \\
\hline Incendios forestales & \\
\hline Lluvias ácidas & \\
\hline Mala gestión de los residuos sólidos ur banos (vertidos incontrolados....) & \\
\hline $\begin{array}{l}\text { Pérdida de tierras cultivables (por crecimiento incontrolado de las ciudades, construcción de } \\
\text { grandes obras públicas, malas prácticas agrícolas....) }\end{array}$ & \\
\hline $\begin{array}{l}\text { Progresivas diferencias Norte-Sur (entre los países desarrollados y los países "en vías de } \\
\text { desarrollo") }\end{array}$ & \\
\hline
\end{tabular}




\section{ANEXO 2}

\section{Cuestionario para la evaluación de la intención de conducta sostenible}

Evalúa tu conducta personal según la escala siguiente:

\begin{tabular}{|l|l|c|c|c|}
\hline \multicolumn{3}{|c|}{ Nunca IT Viempre 3} & \multicolumn{1}{|c}{} \\
\hline 1 & $\begin{array}{l}\text { En mi casa separo en bolsas diferentes los distintos tipos de } \\
\text { basuras }\end{array}$ & 1 & 2 & 3 \\
\hline 2 & $\begin{array}{l}\text { Prescindo de algunas comodidades para proteger el medio } \\
\text { ambiente }\end{array}$ & 1 & 2 & 3 \\
\hline 3 & Compro papel reciclado, aunque es más caro que el normal & 1 & 2 & 3 \\
\hline 4 & $\begin{array}{l}\text { Compro los refrescos envasados en lata porque son más cómodos } \\
\text { de transportar. }\end{array}$ & 1 & 2 & 3 \\
\hline 5 & Utilizo pilas recargables & 1 & 2 & 3 \\
\hline 6 & Cada vez que compro algo pienso en los residuos que genera & 1 & 2 & 3 \\
\hline 7 & Dejo correr el agua del lavabo mientras me cepillo los dientes & 1 & 2 & 3 \\
\hline 8 & Cuando me desplazo en la ciudad utilizo los transportes públicos & 1 & 2 & 3 \\
\hline 9 & Me gusta vestirme con marcas conocidas, aunque vaya "informal". & 1 & 2 & 3 \\
\hline 10 & $\begin{array}{l}\text { Compro alimentos y otros productos elaborados lo más localmente } \\
\text { posible }\end{array}$ & 1 & 2 & 3 \\
\hline 11 & $\begin{array}{l}\text { Me gusta la calefacción alta, para poder estar en mangas de } \\
\text { camisa }\end{array}$ & 1 & 2 & 3 \\
\hline 12 & $\begin{array}{l}\text { Participo en campañas y protestas contra agresiones al medio } \\
\text { ambiente }\end{array}$ & 1 & 2 & 3 \\
\hline 13 & Compro habitualmente productos de "usar y tirar" & 1 & 2 & 3 \\
\hline 14 & $\begin{array}{l}\text { Deposito las botellas en sus contenedores, aunque tenga que } \\
\text { cargar con ellas }\end{array}$ & 1 & 2 & 3 \\
\hline 15 & $\begin{array}{l}\text { Prefiero comprar en grandes superficies, porque encuentro "de } \\
\text { todo" }\end{array}$ & 1 & 2 & 3 \\
\hline 16 & Siempre que puedo me desplazo andando o en bicicleta & 1 & 2 & 3 \\
\hline 17 & No desenchufo los aparatos eléctricos cuando dejo de utilizarlos & 1 & 2 & 3 \\
\hline 18 & Procuro ir a la moda & 1 & 2 & 3 \\
\hline 19 & Dejo las luces encendidas cuando salgo de una habitación & 1 & 2 & 3 \\
\hline 20 & $\begin{array}{l}\text { Compro preferentemente en tiendas que destinan parte de sus } \\
\text { beneficios a los países más necesitados }\end{array}$ & 1 & 2 & 3 \\
\hline
\end{tabular}

Toda a correspondência relativa a este artigo deve ser enviada para: Pedro Álvarez Suárez, Departamento de Didáctica de las Ciencias Experimentales, Facultad de Ciencias de La Educación,Universidad de Granada, 18071 Granada, España. Teléf.: +34-58-240928; Fax: +34-58243555; e-mail: palvarez@ugr.es 